\title{
A case study from the Lynn Sage Comprehensive Cancer Center. Primary sarcoma of the breast: a diagnostic dilemma
}

\author{
J. B. Manders ${ }^{a}$, L. D. Barke ${ }^{\text {b }, ~ A . ~ W a h l ' ~}{ }^{\text {, J. J. A. Wolfman }}{ }^{\text {b }}$, W. Small Jr. ${ }^{c}$, K. M. Keeney ${ }^{a}$, \\ W. J. Gradishard, L. K. Diaz Jr. \\ Departments of a Surgery, ${ }^{b}$ Radiology, ${ }^{c}$ Radiation Oncology, ${ }^{d}$ Medicine, and ${ }^{e}$ Pathology, Northwestern \\ University Feinberg School of Medicine, Lynn Sage Comprehensive Breast Center, \\ Northwestern Memorial Hospital, Chicago, IL, USA.
}

\section{Case report}

A 44-year-old premenopausal, nulliparous woman presented with a recently discovered $4 \mathrm{~cm}$ palpable mass in the upper outer quadrant of her right breast. She denied any skin changes, nipple discharge, or axillary adenopathy. She had no family history of breast cancer and has had no previous mammographic abnormalities or biopsies.

On physical examination, the mass was palpable at the 10 o'clock position of the right breast. There were no nipple or skin changes, no cervical, supraclavicular, or axillary adenopathy, and no expressible nipple fluid.

Diagnostic mammography was performed with radiopaque markers placed at the level of the palpable abnormality, located in the posterior aspect of the right upper outer quadrant and over the palpable mass. Her prior mammograms were performed at an outside institution and were unavailable for comparison.

The film-screen mammographic views revealed a $4.0 \times 3.5 \mathrm{~cm}$ macrolobulated, hyperdense mass corresponding to the palpable area, a portion of which demonstrated ill-defined margins (Fig. 1).

To further characterize the mass, sonography was performed and revealed a complex mass with a near-anechoic portion, suggesting a cystic or necrotic component. In addition, an eccentric $1.2 \mathrm{~cm}$, more

Correspondence to: Leslie K. Diaz, Jr., MD, Assistant Professor of Pathology, Northwestern University Feinberg School of Medicine, 251 E. Huron, Feinberg 7-325, Chicago, IL 60611, USA. E-mail: I-diaz@northwestern.edu; Tel: +1 312926 7405; Fax: +1312 9263127

Publication date 30/06/05 BCO/409/2005/CS echogenic component was identified with increased vascularity within the mass, and increased echogenicity was seen in the surrounding tissue (Fig. 2).

Core needle biopsy of the lesion was performed under ultrasound guidance, consisting of six cores, $0.1 \mathrm{~cm}$ in diameter and ranging in length from 0.6 to $1.2 \mathrm{~cm}$. The cores were entirely composed of a purely high-grade spindle cell proliferation. The differential diagnosis generated from the core biopsy specimen was broad and included: high-grade sarcoma, spindle cell carcinoma, spindle cell malignant melanoma and the sarcomatous component of malignant phyllodes tumor. Smooth muscle actin was focally expressed by tumor cells when assessed by immunohistochemical staining, and the cells were negative for cytokeratin, p63 and melanoma markers. The lesion was interpreted as a malignant spindle cell tumor and a metaplastic carcinoma was favored, however, the possibility of sarcoma could not be excluded.

A wide excision was performed measuring $10 \times 7 \times 4 \mathrm{~cm}$, along with sampling of additional anterior and deep margins. Upon sectioning, a $2.2 \times 2.0 \times 1.0 \mathrm{~cm}$ tan-white, firm, stellate, somewhat ill-defined lesion in the inferior to central portion of the main specimen was identified. The margins were unremarkable. The specimen was extensively sectioned and the lesion was entirely submitted for tissue analysis. Microscopic examination of the hematoxylin- and eosin-stained histologic sections revealed similar morphologic findings to those seen for the needle core biopsy (Fig. 3). The lesion consisted of pleomorphic, high-grade spindle cells with a moderate number of mitosis and focal areas of 


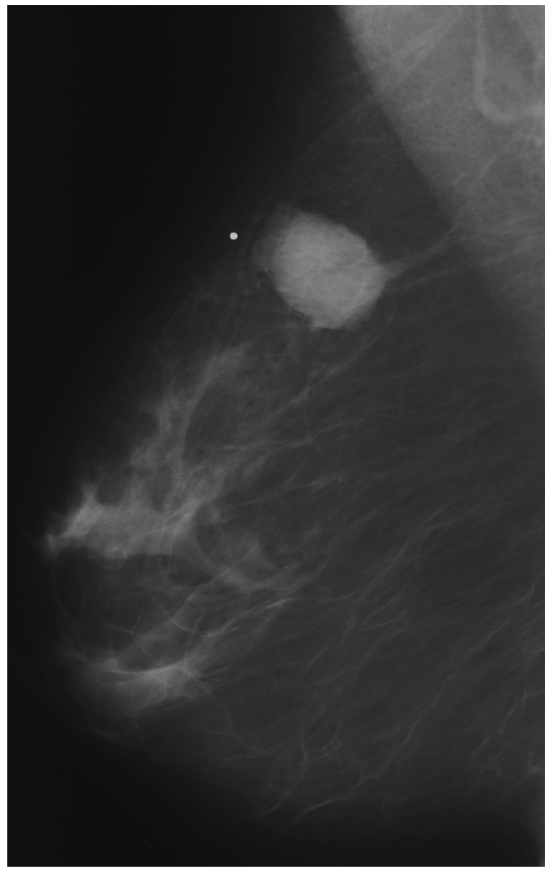

(a)

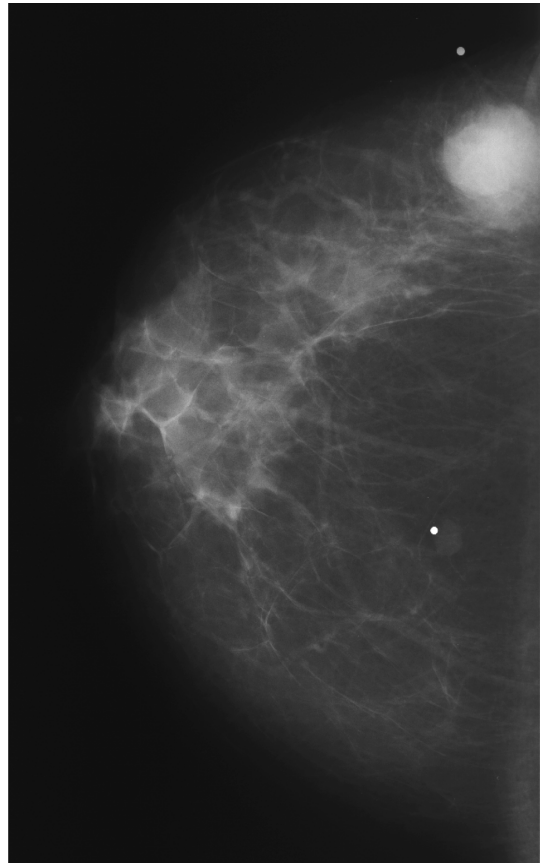

(b)

Figure 1.

(a) Mediolateral oblique and (b) craniocaudal mammograms of the right breast.

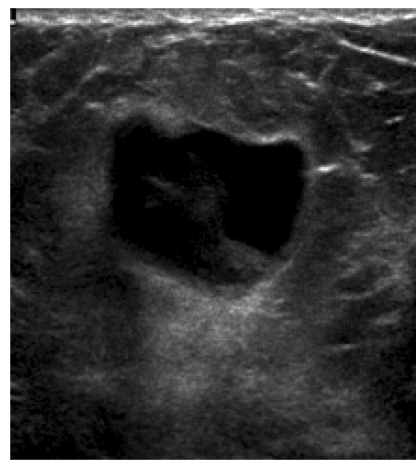

(a)

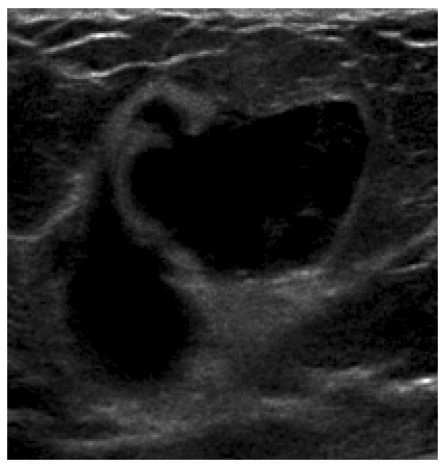

(b)

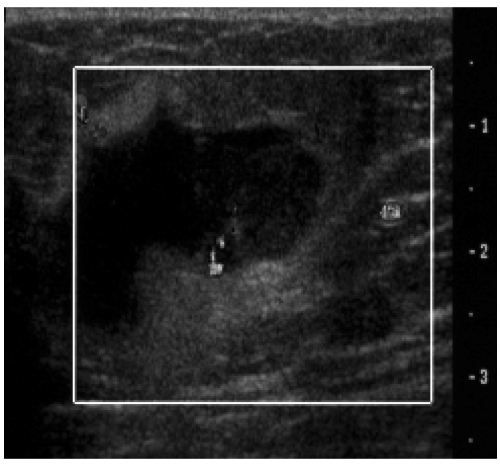

(c)

Figure 2.

Sonographic images of the palpable right breast mass in (a) radial projection, (b) antiradial projection and (c) with the use of color Doppler.

necrosis. These histologic features were definitive for malignancy. The surrounding breast tissue contained fibrocystic changes and areas of lobular carcinoma in situ. No morphologic features that would suggest malignant melanoma or malignant phyllodes tumor were present. Moreover, no evidence of epithelioid cells, squamous differentiation, or ductal carcinoma in situ were identified, thus the lesion was purely a high-grade spindle cell neoplasm.

Immunohistochemical analyses for multiple keratin types, including wide spectrum keratin, consistently yielded completely negative staining in the tumor cells. The absence of epithelioid elements, coupled with the lack of any detectable keratin expression by the tumor cells, prompted a final classification of the lesion as a high-grade pleomorphic sarcoma.

The patient recovered uneventfully from her surgical excision and sentinel lymph node biopsy. Five months have passed since the diagnosis and completion of her treatment. She has not had any disease recurrence and has received no adjuvant radiation or chemotherapy.

Primary breast sarcoma is rare, accounting for less than $1 \%$ of all breast malignancies [1]. Several 


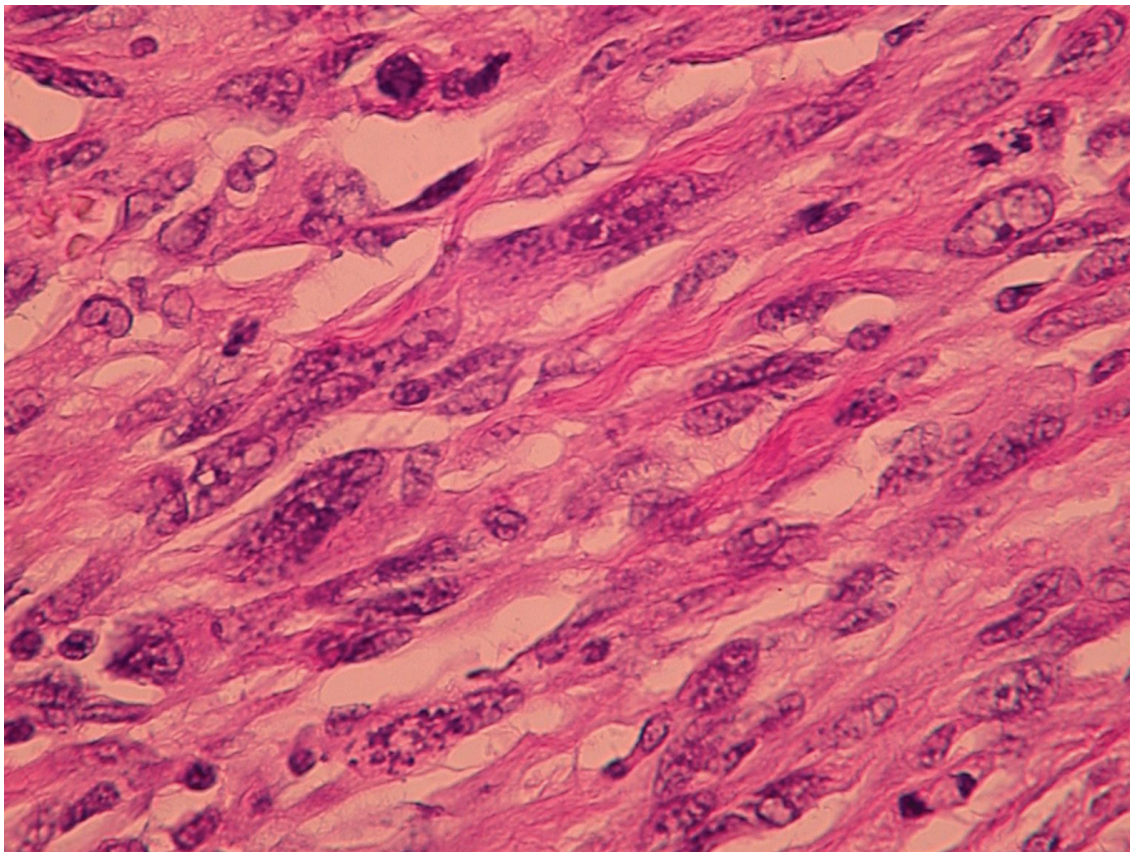

Figure 3.

Hematoxylin and eosin section showing high-grade spindle cells.

histologic subtypes have been described, including malignant fibrous histiocytoma, stromal sarcoma, angiosarcoma, fibrosarcoma, liposarcoma, and others. (Malignant cystosarcoma phyllodes and radiationinduced sarcomas are not grouped with the abovenamed subtypes and are not covered in this discussion.) Recommendations for treatment of this malignancy vary throughout the literature, with some advocating total mastectomy, while others support breast conservation with wide margins $[1,2]$. Local recurrences increase as the tumor size increases, but extent of surgery for the primary lesion does not seem to influence local control as long as negative margins are attained $[1,2]$. Local and distant recurrences and death due to disease are more likely with high-grade lesions. Histologic grade and size are highly predictive of outcome [3].

The significance of this case pertains to its initial pathologic classification. As metaplastic carcinoma was favored initially on the core biopsy, but the possibility of sarcoma could not be excluded, the treatment plan was designed to accommodate the likelihood of either of these diagnoses. Pure spindle cell lesions with malignant features identified by core biopsy should always raise the differential diagnosis of sarcoma, but metaplastic carcinoma must be excluded. This may necessitate the definitive pathologic evaluation to be deferred to the excisional specimen. The identification of epithelioid (carcinomatous) elements or cytokeratin expression by tumor cells is needed for a diagnosis of metaplastic carcinoma to be established [4].

Since metaplastic carcinoma carries with it a risk of lymphatic metastases, a sentinel lymph node biopsy was performed in this case; however, this is not the usual recommendation for sarcoma of the breast. An analysis of 78 patients with primary breast sarcoma without distant metastases at presentation demonstrated rare instances of lymph node metastases $(8 \%)$, however, only one patient with lymph node metastases had sarcoma, and the other two patients actually had carcinosarcoma [2]. A review of the Mayo Clinic experience of primary breast sarcoma demonstrated two patients with metastatically involved lymph nodes out of $22(9.1 \%)$ who had undergone axillary dissections. However, both patients presented with widespread disease [3].

Currently there is no established role for adjuvant systemic chemotherapy in patients with a diagnosis of primary breast sarcoma. Since the diagnosis of primary breast sarcoma is extremely uncommon, there are no data from clinical trials to clearly support the use of adjuvant chemotherapy. At best, small case series and anecdotal reports have described the use of adjuvant chemotherapy, however, there is no compelling evidence that its use is associated with an improved disease-free or overall survival [3]. Similarly, adjuvant radiation therapy following resection of primary breast cancer, particularly in those patients with involved margins, has been advocated 
by some; however, it remains unclear what additional benefit radiation therapy provides [2,3].

Radiotherapy often plays a role in the adjuvant therapy of sarcomas, either to reduce local recurrence and/or provide the opportunity for limb preservation. It would seem logical that radiation would play a similar role in primary sarcomas of the breast. The data is limited, although McGowan et al. suggest that radiation plays a prominent adjuvant role to surgical resection, especially in patients with close or positive margins [2].

In summary, the evaluation of spindle cell lesions by core needle biopsy can be challenging, especially when they are composed purely of spindle cell elements. The possibility of a sarcomatoidtype of metaplastic carcinoma must be excluded.
A multidisciplinary approach to patients with these lesions offers the highest likelihood for cure.

\section{References}

1. Moore MP, Kinne DW. Breast sarcoma. Surg Clin North Am 1996; 76: 383-392.

2. McGowan TS, Cummings BJ, O'Sullivan B, Catton CN, Miller N, Panzarella T. An analysis of 78 breast sarcoma patients without distant metastases at presentation. Int $J$ Radiat Oncol Biol Phys 2000; 46: 383-390.

3. Blanchard DK, Reynolds CA, Grant CS, Donohue JH. Primary nonphylloides breast sarcomas. Am J Surg 2003; 186: 359-361.

4. Brogi E. Benign and malignant spindle cell lesions of the breast. Semin Diagn Pathol 2004; 21: 57-64. 